Section Editor

John J. Millichap, MD

\title{
An isolated cranial nerve 6 palsy as a presentation of polycythemia vera
}

\section{PEARLS}

Aditi Ahlawat, BS, MD

Adalia Jun-O'Connell, MD

Johnny Salameh, MD

Correspondence to

Dr. Salameh:

johnny.salameh@umassmemorial. org
1. Polycythemia vera (PV) is a myeloproliferative disorder that can manifest neurologically with symptoms such as cerebrovascular events and cranial neuropathies involving cranial nerves 3, 4, and 6 .

2. Neurologic sequelae of PV are difficult to diagnose as other conflicting comorbidities may also play a role in the development and identification of symptoms.

\section{OY-STERS}

1. Neurologic manifestations including hemorrhagic or ischemic stroke can occur in patients despite well-controlled disease, with normal hemoglobin and hematocrit levels.

2. Patients with PV and neurologic symptoms remain at high risk for future cerebrovascular events and should be monitored frequently.

Polycythemia vera (PV) is a myeloproliferative disorder associated with neurologic sequelae. The most severe neurologic processes affecting patients with PV are thrombotic and hemorrhagic strokes. ${ }^{1}$ Few cases published in the medical literature describe developments of other neurologic symptoms, including papilledema, oculomotor nerve palsy, ${ }^{2}$ one-and-a-half syndrome, ${ }^{3}$ and trochlear nerve syndrome, secondary to $\mathrm{PV} .{ }^{4}$ One case report describes a cranial nerve 6 abducens palsy in PV due to subarachnoid hemorrhage with concomitant brainstem infarction. ${ }^{5}$ Generally, ophthalmoplegia is a rare complication in PV. We present an unusual case of an isolated abducens nerve palsy in established PV, without evidence of an acute cerebrovascular event on imaging. This patient presentation raises a diagnostic challenge for physicians as the abducens palsy could be attributed to an idiopathic occurrence or PV, the latter of which requires further monitoring and treatment follow-up.

CASE REPORT A 65-year-old left-handed man with a history of chronic PV, stable over 16 years with treatment including folic acid, aspirin, and routine phlebotomy (Q4-6 months), presented to the emergency department with an acute onset of binocular diplopia on right gaze. He reported an associated dull ache surrounding his right orbit, which started a few hours before the diplopia. This event occurred just prior to a scheduled phlebotomy session. His medical history was unremarkable for any trauma, prior stroke, myocardial infarction, or recent infection.

On physical examination, the patient was afebrile with a blood pressure of 150/100 $\mathrm{mm} \mathrm{Hg}$ and a heart rate of 61 beats per minute. Neurologic examination was as follows: the pupils were equal, round, and reactive to light. Funduscopic examination revealed clear optic discs. Although the patient complained of blurred vision, his uncorrected vision was 20/30 R, 20/40 L, and the pinhole test showed 20/20 acuity. Visual fields were normal to double stimulation and confrontation. No ptosis was noted. Extraocular movements were examined and on primary gaze, the right eye was slightly adducted. On horizontal gaze toward the right, the right eye had limited abduction, and the diplopia became more pronounced. Diplopia improved when looking at near objects vs afar. There was no restriction of the patient's gaze toward the left, superiorly, or inferiorly. There was no evidence of an internuclear ophthalmoplegia or nystagmus. The left eye had full extraocular movements. The remaining cranial nerves examined were normal including a symmetric face, normal V1V3 sensation, normal hearing, normal gag reflex, midline tongue, and normal shoulder shrug. No other focal neurologic deficits were noted.

CT head demonstrated mild central atrophy and white matter changes with no acute intracranial abnormalities. CT angiogram was unremarkable except for diffuse irregularity of the left vertebral artery with significant stenosis of the V3 segment, which was likely secondary to a probable chronic dissection. MRI revealed nonspecific fluid-attenuated inversion recovery and T2 white matter hyperintensities, likely representing a chronic small vessel ischemia. An MRI-fast imaging employing steady-state acquisition sequence did not reveal any pathologic findings for the cranial nerves and did not show the presence of a cavernous sinus thrombosis.

From the Department of Neurology, University of Massachusetts Medical School, Worcester.

Go to Neurology.org for full disclosures. Funding information and disclosures deemed relevant by the authors, if any, are provided at the end of the article. 
MRI-susceptibility-weighted angiography (SWAN) sequence showed pronounced susceptibility within the veins, likely related to the known PV (figure).

Pertinent laboratory tests demonstrated hemoglobin of $17.7 \mathrm{~g} / \mathrm{dL}$ and hematocrit of $51.7 \%$, both of which are in the upper limits of normal range. The patient's platelets numbered $232,000 / \mathrm{mm}^{3}$, which is in the normal value range. Other laboratory tests such as the basic metabolic panel, liver enzymes, glycosylated hemoglobin, vitamin $\mathrm{B}_{12}$, coagulation factors, alcohol level, and thyroid function tests were unremarkable. In addition, inflammatory markers such as erythrocyte sedimentation rate, C-reactive protein, Lyme titer, syphilis serology, and angiotensin converting enzyme were all unremarkable.

The major differential diagnosis that could have potentially explained the patient's symptoms included possible small vessel ischemic disease due to the underlying chronic PV or an idiopathic etiology. Although the MRI brain showed minimal small vessel disease changes, it did not reveal obvious small ischemic changes in the brainstem. The extensive investigation did not show any evidence of an acute cause of a sixth nerve palsy. Furthermore, he lacked other cerebrovascular risk factors such as diabetes or hyperlipidemia, with the exception of his treated hypertension. Ophthalmologic evaluation was unremarkable otherwise. After considering both the patient presentation and pertinent diagnostic testing, one plausible explanation for his sixth nerve palsy was a possible neurologic presentation of his underlying myeloproliferative disorder.

\section{Figure Typical radiologic findings in established polycythemia vera}

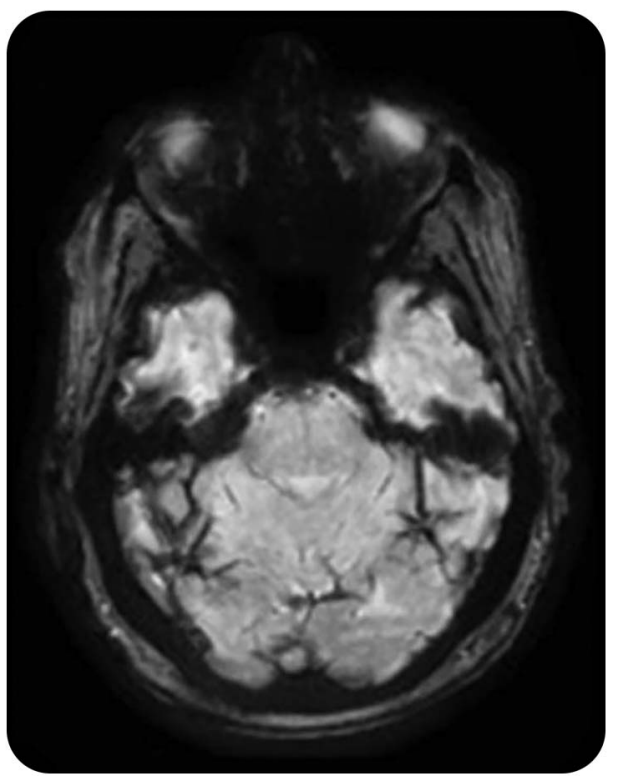

MRI-susceptibility-weighted angiography sequence shows pronounced susceptibility within the veins, likely cerebral venous congestion related to chronic polycythemia vera.
The patient did not require phlebotomy during the hospitalization due to stability of his hemoglobin/hematocrit, and he was already scheduled for an outpatient session upon discharge with his hematologist. He was continued on his routine home medications, including aspirin $81 \mathrm{mg}$ PO daily. The patient applied an eye patch for diplopia, and the symptom resolved within 52 days from the initial onset.

DISCUSSION Patients with PV presenting with neurologic symptoms including cranial neuropathies and stroke represent a challenging diagnostic problem for clinicians. PV is a myeloproliferative disorder known to cause both thrombosis and hemorrhage in the nervous system. ${ }^{1}$ As described above, only a small number of cases have reported cranial neuropathies and ophthalmoplegia in PV. ${ }^{2-5}$ Our case is an unusual presentation of a sixth nerve palsy possibly due to $\mathrm{PV}$, as thrombotic risk is elevated in such patients, or from an idiopathic cause. An extensive investigation excluded other identifiable explanations for the abducens palsy including trauma, infection, inflammatory, and vasculopathic risks (atherosclerosis and diabetes). Discerning whether cranial neuropathies or stroke symptoms arise from well-recognized etiologies, idiopathic events, or PV is difficult and warrants a thorough inquiry to identify treatable causes. In this case, MRI-SWAN imaging demonstrated dilated cerebral veins suggestive of chronic PV. This raised our suspicion that the patient's myeloproliferative disorder may have contributed to his symptoms. Regardless of the cause, recognizing patients with neurologic symptoms in PV is imperative, as this may represent a population at higher risk for cerebrovascular events.

The mechanism of thrombosis in PV is most likely due to high blood viscosity, disseminated intravascular clots, and thrombocytosis. Notably, patients with PV are at higher risk of thromboembolic complications, such as stroke, compared to the general population. ${ }^{6}$ Patients diagnosed with PV have approximately a 10 -fold increase in the risk of thrombotic events, with a $1 \%-1.5 \%$ per year risk vs a $0.1 \%-0.2 \%$ risk in the general population of Western countries. ${ }^{6}$

Although phlebotomy results in reduction of blood viscosity in PV, current evidence suggests that neurologic manifestations of myeloproliferative disorders may not solely occur as a result of increased hyperviscosity alone. Instead, proposed mechanisms in patients with PV causing such varied neurologic symptoms include hypoperfusion from venous stasis and receptor hypersensitivity to catecholamines causing vasoconstriction of the blood supply to the nerve. ${ }^{7,5}$ These mechanisms are difficult to identify via laboratory testing such as complete blood cell 
counts or imaging. Looking forward, developing further tests that utilize these potential mechanisms to better identify those at increased risk for cerebrovascular events will aid in prevention.

Current recommendations for PV treatment include hematocrit control by phlebotomy, as well as myelosuppression agents in those with high thrombosis risk. ${ }^{8}$ Low-dose aspirin is also a part of grade $2 \mathrm{C}$ recommendations in the management of PV. ${ }^{8}$ Lowdose aspirin may be safe and protective in preventing thrombosis due to $\mathrm{PV},{ }^{6}$ as evidence shows that in vivo platelet biochemistry in PV is abnormal. Therefore, low-dose aspirin can suppress the increased thromboxane production. ${ }^{9}$ A Cochrane analysis did not show significant benefit of low-dose aspirin in preventing fatal thrombosis or mortality, however. There was also no association between platelet count and thrombosis, or a predictive value of hematocrit level for thrombosis. ${ }^{10}$

It is important to continue to discern how we can predict the subset of the population that will develop hemorrhagic and thrombotic strokes from chronic polycythemia complications. We raise a potential concern that neurologic complications can occur in patients with well-controlled disease, as in our patient, who potentially experienced thrombotic complications in spite of maximal recommended treatments within the appropriate ranges of hematocrit and platelet levels. Recognition of neurologic sequelae even in wellcontrolled patients with PV is an important indicator of disease progression and prompts the clinician to reassess treatment options and risk stratification. In this case, although the patient's cranial neuropathy resolved, he was targeted as a high-risk patient for a possible future cerebrovascular event and treatment was tailored to control his risk factors.

\section{AUTHOR CONTRIBUTIONS}

Aditi Ahlawat: drafting/revising the manuscript, analysis or interpretation of data, accepts responsibility for conduct of research and final approval, contribution of vital reagents/tools/patients, acquisition of data. Adalia
Jun-O'Connell: drafting/revising the manuscript, accepts responsibility for conduct of research and final approval, study supervision. Johnny S. Salameh: drafting/revising the manuscript, study concept or design, analysis or interpretation of data, accepts responsibility for conduct of research and final approval, study supervision.

\section{STUDY FUNDING}

No targeted funding reported.

\section{DISCLOSURE}

The authors report no disclosures relevant to the manuscript. Go to Neurology.org for full disclosures.

\section{REFERENCES}

1. Tefferi A. Polycythemia vera and essential thrombocythemia: 2013 update on diagnosis, risk-stratification, and management. Am J Hematol 2013;88:508-516.

2. Park YH, Huh YE, Kim JS. Case report: oculomotor nerve palsy as an initial manifestation of polycythemia vera. J Clin Neurosci 2012;19:328-330.

3. Lousa M, Gobernado JM, Gimeno A. One-and-a-half syndrome due to polycythemia. J Neurol Neurosurg Psychiatry $1983 ; 46: 873$.

4. Jones M, Colin C, Rowe D. Isolated trochlear nerve palsy as a presenting feature of primary polycythemia rubra vera. Clin Exp Ophthalmol 2004;32:339-340.

5. Opherk C, Bruning R, Pellkofer HL, Dichgans M, Hamann GF. Subarachnoid hemorrhage and diplopia as initial presentation of polycythemia vera. Cerebrovasc Dis 2005; 19:279-280.

6. Patrono C, Rocca B, De Stefano V. Platelet activation and inhibition in polycythemia vera and essential thrombocythemia. Blood 2013;121:1701.

7. Tarach JS, Tarach BM. Myeloproliferative disorders: neurological complications. Med Sci Monit 2000;6:421-425.

8. Tefferi A. Prognosis and treatment of polycythemia vera. In: UpToDate [online]. Available at: http://www.uptodate. com/contents/prognosis-and-treatment-of-polycythemia-vera. Accessed October 5, 2014.

9. Landolfi R, Ciabattoni G, Patrignani P, et al. Increased thromboxane biosynthesis in patients with polycythemia vera: evidence for aspirin-suppressible platelet activation in vivo. Blood 1992;80:1965.

10. Squizzato A, Romualdi E, Passamonti F, Middeldorp S. Antiplatelet drugs for polycythemia vera and essential thrombocythemia. Cochrane Database Syst Rev 2013;4: CD006503. 


\section{Neurology}

\section{Pearls \& Oy-sters: An isolated cranial nerve 6 palsy as a presentation of polycythemia vera \\ Aditi Ahlawat, Adalia Jun-O'Connell and Johnny Salameh \\ Neurology 2015;85;e85-e87 \\ DOI 10.1212/WNL.0000000000001927}

This information is current as of September 14, 2015

Updated Information \& Services

References

Permissions \& Licensing

Reprints including high resolution figures, can be found at: http://n.neurology.org/content/85/11/e85.full

This article cites 9 articles, 3 of which you can access for free at: http://n.neurology.org/content/85/11/e85.full\#ref-list-1

Information about reproducing this article in parts (figures,tables) or in its entirety can be found online at:

http://www.neurology.org/about/about_the_journal\#permissions

Information about ordering reprints can be found online: http://n.neurology.org/subscribers/advertise

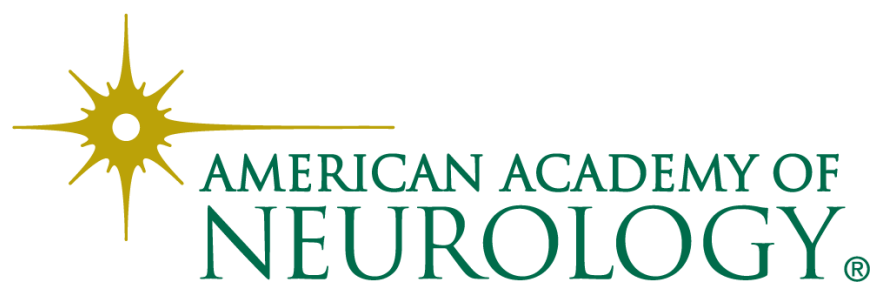

